\title{
Dual-Genotype Orientia tsutsugamushi Infection in Patient with Rash and Eschar, Vietnam, 2016
}

\section{Nhiem Le-Viet, Duc-Tuan Phan, Nho Le-Viet, Sinh Trinh, Muoi To, Didier Raoult, Philippe Parola}

We report a dual-genotype Orientia tsutsugamushi infection in Vietnam in 2016. The patient had fever, rash, and an eschar. The Kawasaki genotype was identified in the eschar specimen and Karp genotype in the whole blood specimen. The genotype co-infection rate for scrub typhus is unknown and should be further evaluated.

Crub typhus is an acute febrile zoonosis caused by OriSentia tsutsugamushi that is transmitted by larval trombiculid mites in rural areas (1). Scrub typhus is a major public health issue in the Asia-Pacific region and might also be present in Africa (2) and South America (3). Clinical manifestations can vary from mild symptoms to fatal disease in the absence of appropriate antimicrobial drugs (4). Many antigenic variants of $O$. tsutsugamushi exist, including Gilliam, Kato, Karp, Kawasaki, and Kuroki. This antigenic variation depends largely on the immune-dominant $56-\mathrm{kDa}$ type-specific antigen (TSA) located on the surface of the bacteria membrane. Genotypes of $O$. tsutsugamushi are based on the 56-kDa TSA gene, which are commonly used to identify the diverse strains present in endemic countries (4-6). We report a case of a patient infected with 2 distinct genotypes of $O$. tsutsugamushi.

\section{The Study}

A 35-year-old man, who often harvested agarwood in the forests of central Vietnam, had a fever and headache during a harvesting trip. On the seventh day of illness, he sought treatment at Quang Nam Northern Mountainous Region General Hospital (Quang Nam, Vietnam). He had continuous high fever, rigor, increasing headache, and muscle

Author affiliations: Quang Nam Central General Hospital, Quang Nam, Vietnam (Nhiem Le-Viet, Nho Le-Viet); Aix-Marseille University, IRD, AP-HM, SSA, VITROME, IHU-Méditerranée Infection, Marseille, France (Nhiem Le-Viet, D. Raoult, P. Parola); Quang Nam Northern Mountainous Region General Hospital, Quang Nam (D.-T. Phan, S. Trinh, M. To); Danang University, Danang, Vietnam (Nho Le-Viet)

DOI: https://doi.org/10.3201/eid2408.171622 pain but no nausea, vomiting, or abdominal pain. A rash developed 1 day after the fever. During his time working in the forest, the man took 3 pills of acetaminophen per day to treat his symptoms, but this medicine did not improve his condition. On examination, the patient was highly febrile $\left(39^{\circ} \mathrm{C}-40^{\circ} \mathrm{C}\right)$ and had an oval-shaped, painless eschar measuring $8 \times 10 \mathrm{~mm}$ on his right anterior neck (Figure 1). He also had a rash covering his whole body (Figure 2). Swollen lymph nodes were observed along the mid-jugular chain of the right neck and right armpit; the largest lymph node was $10 \mathrm{~mm}$ in diameter, mobile, and painless. Results of routine laboratory tests for serum aspartate aminotransferase, alanine aminotransferase, and creatinine and complete blood cell counts were within reference ranges. Oral doxycycline $(100 \mathrm{mg})$, prescribed immediately after scrub typhus was suspected, was administered twice a day starting on the first day of hospitalization. Defervescence occurred 24 hours after the first dose of doxycycline, and the man completely recovered.

On the day of admission, we collected a sample of the eschar by rotating a swab vigorously at the eschar base after the crust was removed, as previously described (7). We also took $500 \mu \mathrm{L}$ of whole blood and $500 \mu \mathrm{L}$ of acute-phase plasma and stored these specimens at $-20^{\circ} \mathrm{C}$. Specimens were transported to Marseille, France, for molecular biology and serologic testing.

We isolated DNA from the eschar and whole blood specimens and performed a real-time quantitative PCR (qPCR) specific for the periplasmic serine protease gene of $O$. tsutsugamushi; qPCR results showed these specimens were positive for the protease gene (6). Then, we subjected the DNA to a conventional PCR targeting the $O$. tsutsugamushi $56-\mathrm{kDa}$ TSA gene using appropriate negative controls and sequenced the PCR products. We analyzed the partial 56-kDa TSA gene sequences from our patient with ABI PRISM DNA Sequencing Analysis software version 3.0 (Applied Biosystems, Foster City, CA, USA) and compared them with those available in the GenBank database using the BLAST algorithm (http://blast.ncbi.nlm.nih.gov/ Blast.cgi). The DNA sequence obtained from the eschar specimen (GenBank accession no. MF769529) was closely related to the Kawasaki genotype, showing $97.2 \%$ identity to the reference strain TPC0707a (GenBank accession no. GQ332758) (8). The eschar specimen sequence was even 
more similar to a Kawasaki isolate previously detected in a patient in Quang Nam Province, Vietnam (GenBank accession no. KU871388, 98.8\% identity) (6). The DNA sequence obtained from the whole blood specimen (GenBank accession no. MF769530) demonstrated $98.8 \%$ identity to that of a Karp isolate in Cambodia (GenBank accession no. HQ718422) (4). The whole blood specimen sequence was also highly similar ( $98.4 \%$ identity) to the sequences of 2 Karp-related isolates previously detected in patients in Quang Nam Province (GenBank accession nos. KU871384 and KU871378) (6). We tested the acute-phase plasma sample collected from the patient on the seventh day of illness using a micro-immunofluorescence assay (IFA) that included whole-cell antigens of $O$. tsutsugamushi serotypes Karp, Kato, and Gilliam. However, results were negative for specific antibodies to these antigens.

We tested for dengue virus, Plasmodium spp., and Leptospira spp. by qPCR using the whole blood specimens; negative results led to the exclusion of these pathogens as the causative agent of the fever. We also tested for other rickettsiae (Rickettsia typhi, R. felis, R. conorii and other spotted fever group rickettsiae, and Coxiella burnetii) using specific qPCRs and IFAs and excluded these pathogens as well.

Because this patient's signs and symptoms included eschar and rash, his clinical presentation was typical for scrub typhus. A rash is observed in $\approx 50 \%$ of patients infected with scrub typhus (9), the percentage varying depending on the O. tsutsugamushi genotype (6). Eschar is associated with many rickettsial diseases and is present in $7 \%-80 \%$ of scrub typhus patients (10). Eschar is associated

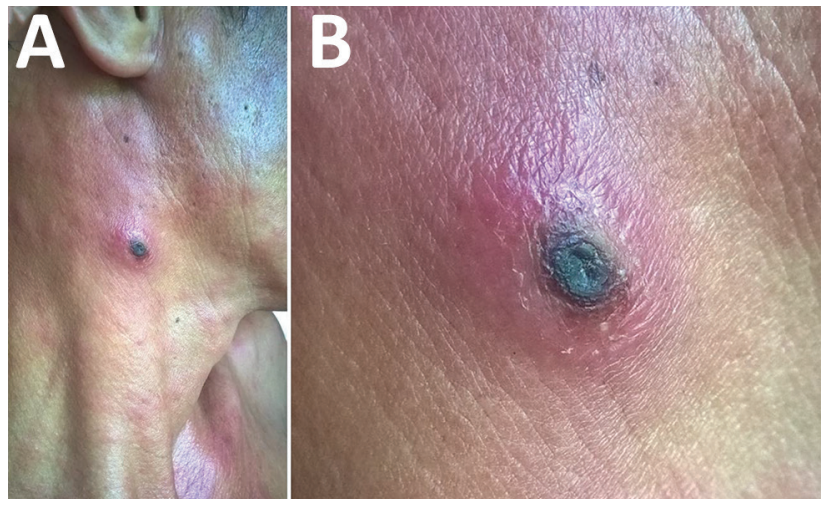

Figure 1. Eschar on right anterior neck of patient with dual genotype Orientia tsutsugamushi infection, Vietnam. A) Eschar location; B) enlarged view.

with severe renal, hematologic, respiratory, and circulatory manifestations; long hospital stay; and high mortality rate (11). The eschar is the preferred sampling site for $O$. tsutsugamushi detection and isolation. Eschar swabs are preferred over eschar biopsy and blood samples because swabbing eschars is noninvasive, easy, and painless and PCRs of DNA from eschar swab samples are highly sensitive and specific $(6,12)$.

In 2014, O. tsutsugamushi genotype co-infections were reported in rodents and wild chiggers, as well as in naturally infected and laboratory-reared mites, in Thailand; the Karp genotype was detected in all mites examined, and Gilliam and UT302 were the co-infecting genotypes (13). Sonthayanon et al. tested whether scrub

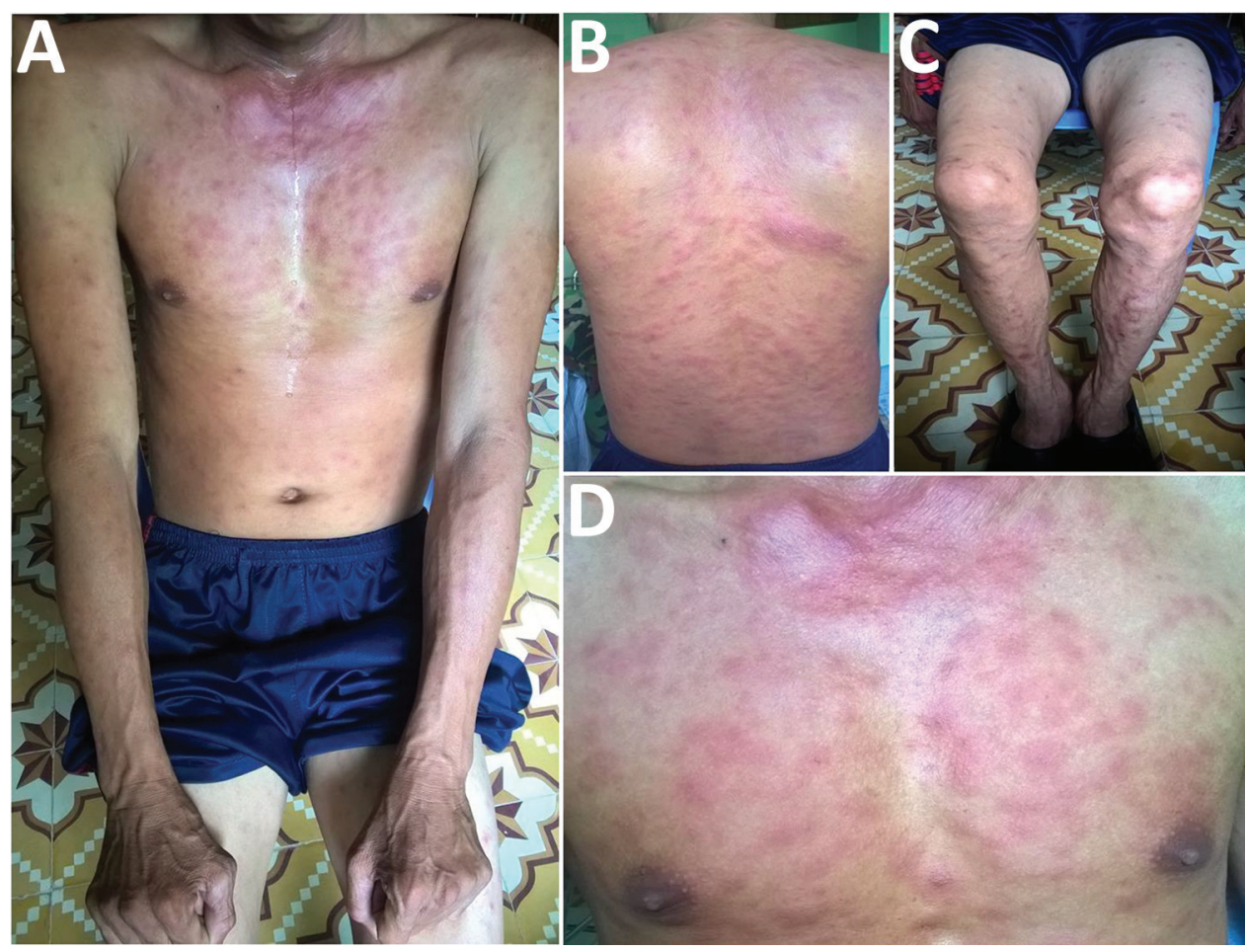

Figure 2. Rash on patient with dual-genotype Orientia tsutsugamushi infection, Vietnam. A) Trunk and arms; B) back; C) legs; D) chest. 
typhus patients were simultaneously infected with multiple $O$. tsutsugamushi genotypes using multilocus sequence typing (14); however, the specific genotypes could not be identified in their study.

\section{Conclusions}

We demonstrated the coexistence of 2 different $O$. tsutsugamushi genotypes in this patient. The negative $O$. tsutsugamushi IFA results could have been a result of early stage plasma collection, on the seventh day of illness. For efficient serologic diagnosis, convalescent-phase plasma or serum is needed. Unfortunately, we could not obtain this type of sample from this patient because he returned to work in the forest after 5 days of treatment. Because serologic results can be negative for over a week after scrub typhus disease onset, we stress use of molecular assays rather than serologic tests for diagnosis.

We used appropriate controls and obtained sequences that had not been obtained before in our laboratory, making contamination a highly unlikely explanation for our results. However, in vitro isolation would have been stronger evidence for a dual $O$. tsutsugamushi infection.

Because this patient often worked in the forest, his dual infection could have been caused by bites from several mites, but bites from a single mite infected with multiple genotypes could have also happened $(13,14)$. Although detecting both genotypes in the eschar sample would have been expected if he was bitten by a mite infected with multiple genotypes, 1 genotype might have predominated. Furthermore, a person bitten by multiple mites might have multiple eschars, but eschar formation varies, depending on the $O$. tsutsugamushi genotype (15). Wearing adequate personal protective gear when in wooded areas can help minimize exposure to mites and prevent scrub typhus. However, when a patient such as the one we describe seeks treatment, clinicians should administer doxycycline immediately.

\section{Acknowledgments}

We thank the staff of the Department of Internal Medicine II of Quang Nam Northern Mountainous Region General Hospital, Quang Nam, Vietnam, for their support with sample collection. We also thank the biomol team of Institut Hospitalo-Universitaire Méditerranée Infection, Marseille, France, for their support with serologic testing.

Sample collection in this study was funded by Quang Nam Provincial People's Committee under Provincial Scientific and Technological Tasks (reference no. 19/HD-SKHCN/2016) managed by Quang Nam Department of Science and Technology. Laboratory analyses and publication were funded by the government of France under the Investments of Futures Program (reference no. Méditerranée Infection 10-IAHU-03) managed by the National Agency for Research.

\section{About the Author}

Dr. Nhiem Le-Viet is an infectious diseases clinician at the Quang Nam Central General Hospital in Quang Nam, Vietnam. His research interests are in deciphering the ecology of rickettsial disease and etiologies of acute undifferentiated fevers of Vietnam by using emerging tools.

\section{References}

1. Parola P, Raoult D. Tropical rickettsioses. Clin Dermatol. 2006; 24:191-200. http://dx.doi.org/10.1016/j.clindermatol.2005. 11.007

2. Ghorbani RP, Ghorbani AJ, Jain MK, Walker DH. A case of scrub typhus probably acquired in Africa. Clin Infect Dis. 1997;25: 1473-4. http://dx.doi.org/10.1086/516990

3. Weitzel T, Dittrich S, López J, Phuklia W, Martinez-Valdebenito C, Velásquez K, et al. Endemic scrub typhus in South America. N Engl J Med. 2016;375:954-61. http://dx.doi.org/10.1056/ NEJMoa1603657

4. Duong V, Mai TT, Blasdell K, Lo V, Morvan C, Lay S, et al. Molecular epidemiology of Orientia tsutsugamushi in Cambodia and Central Vietnam reveals a broad region-wide genetic diversity. Infect Genet Evol. 2013;15:35-42. http://dx.doi.org/10.1016/ j.meegid.2011.01.004

5. Varghese GM, Janardhanan J, Mahajan SK, Tariang D, Trowbridge P, Prakash JA, et al. Molecular epidemiology and genetic diversity of Orientia tsutsugamushi from patients with scrub typhus in 3 regions of India. Emerg Infect Dis. 2015;21:64-9. http://dx.doi.org/10.3201/eid2101.140580

6. Le Viet N, Laroche M, Thi Pham HL, Viet NL, Mediannikov O, Raoult D, et al. Use of eschar swabbing for the molecular diagnosis and genotyping of Orientia tsutsugamushi causing scrub typhus in Quang Nam Province, Vietnam. PLoS Negl Trop Dis. 2017;11:e0005397. http://dx.doi.org/10.1371/ journal.pntd.0005397

7. Mouffok N, Socolovschi C, Benabdellah A, Renvoise A, Parola P, Raoult D. Diagnosis of rickettsioses from eschar swab samples, Algeria. Emerg Infect Dis. 2011;17:1968-9. http://dx.doi.org/ 10.3201/eid1710.110332

8. Lu HY, Tsai KH, Yu SK, Cheng CH, Yang JS, Su CL, et al. Phylogenetic analysis of 56-kDa type-specific antigen gene of Orientia tsutsugamushi isolates in Taiwan. Am J Trop Med Hyg. 2010;83:658-63. http://dx.doi.org/10.4269/ ajtmh.2010.09-0608

9. Taylor AJ, Paris DH, Newton PN. A systematic review of mortality from untreated scrub typhus (Orientia tsutsugamushi). PLoS Negl Trop Dis. 2015;9:e0003971. http://dx.doi.org/10.1371/ journal.pntd.0003971

10. Rajapakse S, Rodrigo C, Fernando D. Scrub typhus: pathophysiology, clinical manifestations and prognosis. Asian Pac J Trop Med. 2012;5:261-4. http://dx.doi.org/10.1016/ S1995-7645(12)60036-4

11. Chauhan V, Thakur A, Thakur S. Eschar is associated with poor prognosis in scrub typhus. Indian J Med Res. 2017;145:693-6.

12. Kim DM, Kim HL, Park CY, Yang TY, Lee JH, Yang JT, et al. Clinical usefulness of eschar polymerase chain reaction for the diagnosis of scrub typhus: a prospective study. Clin Infect Dis. 2006;43:1296-300. http://dx.doi.org/10.1086/508464

13. Takhampunya R, Tippayachai B, Promsathaporn S, Leepitakrat S, Monkanna T, Schuster AL, et al. Characterization based on the 56-kDa type-specific antigen gene of Orientia tsutsugamushi genotypes isolated from Leptotrombidium mites and the rodent host post-infection. Am J Trop Med Hyg. 2014;90:139-46. http://dx.doi.org/10.4269/ajtmh.13-0393

14. Sonthayanon P, Peacock SJ, Chierakul W, Wuthiekanun V, Blacksell SD, Holden MT, et al. High rates of homologous 
recombination in the mite endosymbiont and opportunistic human pathogen Orientia tsutsugamushi. PLoS Negl Trop Dis. 2010;4:e752. http://dx.doi.org/10.1371/ journal.pntd.0000752

15. Kim DM, Yun NR, Neupane GP, Shin SH, Ryu SY, Yoon HJ, et al. Differences in clinical features according to Boryoung and Karp genotypes of Orientia tsutsugamushi.
PLoS One. 2011;6:e22731. http://dx.doi.org/10.1371/ journal.pone. 0022731

Address for correspondence: Philippe Parola, Vitrome, Institut HospitaloUniversitaire Méditerranée Infection, 19-21 Blvd Jean Moulin 13385, Marseille CEDEX 05, France; email: philippe.parola@univ-amu.fr

\section{August 2017: Vectorborne Infections}

- Added Value of Next-Generation Sequencing for Multilocus Sequence Typing Analysis of a Pneumocystis jirovecii Pneumonia Outbreak

- Bartonella quintana, an Unrecognized Cause of Infective Endocarditis in Children in Ethiopia

- Characteristics of Dysphagia in Infants with Microcephaly Caused by Congenital Zika Virus Infection, Brazil, 2015

- Zika Virus Infection in Patient with No Known Risk Factors, Utah, USA, 2016

- Acute Febrile Illness and Complications Due to Murine Typhus, Texas, USA

- High Infection Rates for Adult Macaques after Intravaginal or Intrarectal Inoculation with Zika Virus

- Lyme Borreliosis in Finland, 1995-2014

- Characterization of Fitzroy River Virus and Serologic Evidence of Human and Animal Infection

- Genomic Characterization of Recrudescent Plasmodium malariae after Treatment with Artemether/Lumefantrine

- Molecular Characterization of Corynebacterium diphtheriae Outbreak Isolates, South Africa, March-June 2015

- Clinical Laboratory Values as Early Indicators of Ebola Virus Infection in Nonhuman Primates

- Maguari Virus Associated with Human Disease

- Human Infection with Highly Pathogenic Avian Influenza $\mathrm{A}(\mathrm{H} 7 \mathrm{~N} 9)$ Virus, China

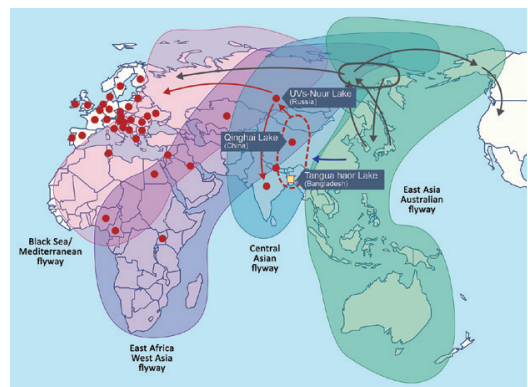

- Serologic Evidence of Scrub Typhus in the Peruvian Amazon

- Global Spread of Norovirus Gll. 17 Kawasaki 308, 2014-2016

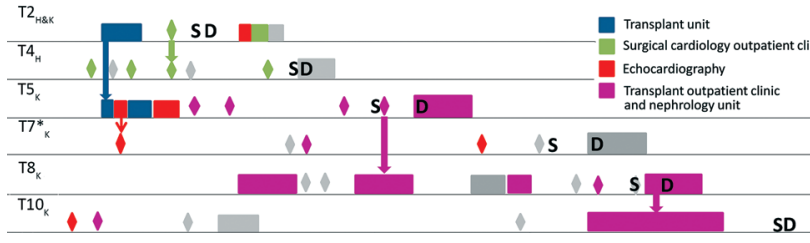

- Preliminary Epidemiology of Human Infections with Highly Pathogenic Avian Influenza A(H7N9) Virus, China, 2017

- Real-Time Evolution of Zika Virus Disease Outbreak, Roatán, Honduras

- Clonal Expansion of New Penicillin-Resistant Clade of Neisseria meningitidis Serogroup W Clonal Complex 11, Australia

- Genesis of Influenza A(H5N8) Viruses

- Density-Dependent Prevalence of Francisella tularensis in Fluctuating Vole Populations, Northwestern Spain

- Occupational Exposures to Ebola Virus in Ebola Treatment Center, Conakry, Guinea

- West Nile Virus Outbreak in Houston and Harris County, Texas, USA, 2014

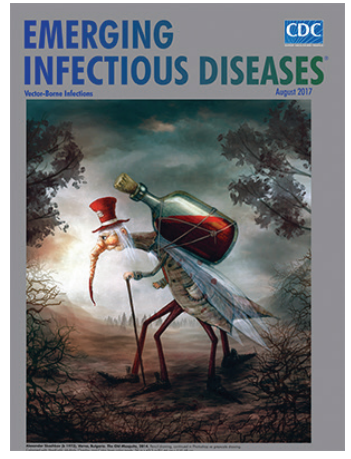

- Human Metapneumovirus and Other Respiratory Viral Infections during Pregnancy and Birth, Nepal

- Serologic Evidence of Powassan Virus Infection in Patients with Suspected Lyme Disease

- Influenza D Virus in Animal Species in Guangdong Province, Southern China

- Seroprevalence of Baylisascaris procyonis Infection among Humans, Santa Barbara County, California, USA, 2014-2016

- Opiate Injection-Associated Skin, Soft Tissue, and Vascular Infections, England, UK, 1997-2016 